BMJ Open Diabetes Research \& Care

\title{
Diabetes and glucose disturbances in patients with psychosis in Sweden
}

\author{
Eric Olsson, ${ }^{1,2}$ Jeanette Westman, ${ }^{3}$ Dzana Sudic Hukic, ${ }^{2,4}$ Sven V Eriksson, ${ }^{5}$ \\ Gunnar Edman, ${ }^{3,4,6}$ Robert Bodén, ${ }^{7,8}$ Erik Jedenius, ${ }^{1}$ Johan Reutfors, ${ }^{7}$ \\ Anders Berntsson, ${ }^{2}$ Agneta Hilding, ${ }^{9}$ Martin Schalling, ${ }^{4,9}$ Claes-Göran Östenson, ${ }^{9}$ \\ Urban Ösby ${ }^{3,4,6}$
}

To cite: Olsson E, Westman J, Sudic Hukic D, et al. Diabetes and glucose disturbances in patients with psychosis in Sweden. BMJ Open Diabetes Research and Care 2015;3:e000120. doi:10.1136/bmjdrc-2015000120

Received 31 May 2015 Revised 31 July 2015 Accepted 25 August 2015

\section{(1) CrossMark}

For numbered affiliations see end of article.

Correspondence to Dr Eric Olsson; eric.olsson@primavuxen.se

\section{ABSTRACT}

Objective: The objectives of this study were to (1) analyze the prevalence of diabetes, prediabetes, and antidiabetic medication in patients with psychosis compared with control subjects and (2) determine what factors in patients with psychosis were associated with antidiabetic medication.

Method: We studied 977 patients with psychosis recruited from outpatient clinics in Stockholm County, Sweden, and they were compared with 3908 nonpsychotic control subjects for fasting plasma glucose levels; prevalence of diabetes, prediabetes, antidiabetic treatment, and tobacco use; and blood pressure, weight, height, and waist circumference. Group differences were evaluated with analysis of variance and $\chi^{2}$ test, and factors associated with antidiabetic treatment were evaluated with logistic regression.

Results: Diabetes was observed in $94(10 \%)$ patients with psychosis, 2.7 times the prevalence observed in control subjects. Among patients with psychosis, $87(10 \%)$ had prediabetes (fasting glucose, 6.1-6.9 mmol/L) compared with $149(3.8 \%)$ control subjects. Most patients with psychosis $(77 \%)$ who had prediabetes fulfilled criteria for metabolic syndrome. In patients with psychosis, both lipid-lowering medication and fasting glucose were significantly associated with antidiabetic treatment. There was no significant relation between antidiabetic treatment and lifestyle factors such as smoking or degree of psychiatric illness.

Conclusions: The high prevalence of impaired fasting glucose and metabolic syndrome in patients with psychosis warrants further clinical research in preventing or delaying the onset of diabetes in these patients by pharmacotherapy and/or lifestyle intervention.

\section{INTRODUCTION}

Severe mental illnesses including schizophrenia and other psychotic disorders are associated with marked excess somatic morbidity, resulting in a twofold increased risk of cardiovascular mortality in patients who have severe mental illnesses compared with the general population. ${ }^{1-3}$ Although suicide is prevalent in these patients, ischemic heart disease is the major contributor to excess

\section{Key messages}

- Draws attention to the high prevalence of prediabetes and diabetes among patients with psychosis.

- Points out that most patients with psychosis with prediabetes also fulfil criteria for metabolic syndrome.

- Proposes that reduced progression to diabetes should be an important treatment goal, in patients with psychosis and that prospective randomized treatment studies should be performed with lifestyle interventions and pharmacological treatment to determine the most effective preventive treatments.

mortality. ${ }^{4-6}$ Diabetes is associated with a several-fold increased risk of developing cardiovascular disease and may contribute to the increased cardiovascular morbidity and mortality in these patients. ${ }^{7}$ In patients with schizophrenia and other types of psychoses, there is an increased risk of diabetes and intermediate stages of glycemic abnormalities. ${ }^{8}$ The primary reason for the increased frequency of type 2 diabetes observed in association with psychosis is unclear, ${ }^{10}{ }^{11} \mathrm{but}$ common genetic factors, lifestyle factors, and treatment with antipsychotic medications may contribute.

The main focus when treating severe mental illness has been to address psychiatric symptoms, and somatic symptoms may be underdiagnosed and less aggressively monitored, potentially contributing to the different frequency of mortality between patients with mental illnesses and the general population. ${ }^{3}$ Broadening the focus of treatment in psychotic disorders to include somatic illness, such as glucose abnormalities, may potentially improve the prognosis. Antidiabetic medications and structured lifestyle interventions may prevent or delay the development of diabetes in people with impaired glucose tolerance. ${ }^{12}$ However, despite the increased risk of diabetes and cardiovascular mortality 
in patients with psychosis, there is little information available about the prevalence of impaired glucose tolerance or the effects of antidiabetic treatment in these patients.

The purposes of this study were to evaluate the prevalence of diabetes, prediabetes (defined as impaired fasting glucose), and antidiabetic treatment in patients with psychosis compared with control subjects, and to determine what factors in patients with psychosis are associated with antidiabetic pharmacological treatment.

\section{MATERIALS AND METHODS}

\section{Patients with psychotic disorders}

Study patients with psychosis were recruited during the years 2005-2010 in the Swedish Study of Metabolic Risks in Psychosis (SMRP) performed in psychosis outpatient clinics primarily in Stockholm County (75\% patients), Sweden. These clinics were responsible for the treatment of all outpatients with long-term psychotic disorders in the catchment area. As part of the general health screening, all patients in regular clinical treatment from each clinic were asked to participate in the study. Psychiatric diagnoses were confirmed by the treating psychiatrist according to published criteria. ${ }^{13}$ In the present analysis, 977 patients with psychosis were included in the study, and family history of diabetes was present in 184 (24\%) patients with psychosis.

\section{Control subjects}

Control subjects were 3908 in a follow-up study of a population-based survey, the Stockholm Diabetes Prevention Program (SDPP). The baseline SDPP study was performed from 2003 to $2006 .{ }^{14}$ The follow-up SDPP study was performed 8-10 years after baseline. Since an aim of the baseline SDPP study was to evaluate the effect of family history of diabetes on diabetes risk, the study sample was selected from all responders and enriched with regard to family history of diabetes, and half the participants had a family history of diabetes, defined as $\geq 1$ first-degree relative with known diabetes. Excluded from the follow-up study were participants who had newly diagnosed diabetes during the baseline health examination, including an oral glucose tolerance test, and together with participants with known diabetes before baseline they comprised $3.7 \%$ of all participants. The follow-up study included 5712 participants, $72 \%$ of the baseline sample. For the present study, 3908 (out of 5712) control subjects were randomly selected to match patients with psychosis with regard to frequency of family history of diabetes. Thus, 956 (24\%) SDPP control subjects included in this study had a family history of diabetes.

\section{Procedures}

Patients were assessed with a psychiatric questionnaire that included diagnosis, level of functioning (assessed with Global Assessment of Functioning scale), severity of mental illness (assessed with Clinical Global Impression scale), duration of illness, and duration of treatment. ${ }^{15} 16$ For patients with psychosis and control subjects, all present medications and dosages were recorded. In patients with psychosis, current medication was confirmed in hospital records. Patients with psychosis and control subjects were asked about diabetes in first-degree relatives. For patients with psychosis and control subjects, somatic health was assessed with a questionnaire about diabetes and hypertension. Both patients with psychosis and control subjects were asked about current tobacco use, and blood pressure, body mass index, and waist circumference were measured. Patients with psychosis and control subjects were given written instructions to fast overnight before venous blood sampling.

\section{Assays}

Fasting plasma glucose (FPG) was measured in patients with psychosis and control subjects. All control subjects without diagnosed diabetes did an oral glucose tolerance test. Lipid profiles, including total cholesterol, high-density lipoprotein (HDL), low-density lipoprotein (LDL), and triglycerides, were determined for all patients with psychosis but not for control subjects by the Karolinska University Laboratory.

\section{Metabolic syndrome, diabetes and prediabetes}

The metabolic syndrome includes the most important risk factors for cardiovascular disease: diabetes and impaired fasting glucose, abdominal obesity, dyslipidemia, and hypertension. Metabolic syndrome was defined according to the International Diabetes Federation (IDF) criteria: increased waist circumference (ethnicityspecific values: $\geq 94 \mathrm{~cm}$ in male and $\geq 80 \mathrm{~cm}$ in female European Caucasians) plus any two of the following four factors: elevated triglycerides $(\geq 1.7 \mathrm{mmol} / \mathrm{L})$, reduced HDL cholesterol $(<1.03 \mathrm{mmol} / \mathrm{L}$ in males and $<1.29 \mathrm{mmol} / \mathrm{L}$ in females), elevated blood pressure (systolic $\geq 130 \mathrm{~mm} \mathrm{Hg}$, diastolic $\geq 85 \mathrm{~mm} \mathrm{Hg}$, or antihypertensive treatment), and elevated FPG $(\geq 5.6 \mathrm{mmol} / \mathrm{L}$ or antidiabetic drug treatment). ${ }^{17-19}$ Metabolic syndrome could be determined only in patients with psychosis because lipid profiles were only available in these patients. The definitions of the WHO and IDF were used for the prediabetic state (fasting glucose, $6.1-6.9 \mathrm{mmol} / \mathrm{L}$ ) and diabetes (fasting glucose $\geq 7.0 \mathrm{mmol} / \mathrm{L}$ ). ${ }^{20}$ The American Diabetes Association definition of impaired fasting glucose was used (fasting glucose, $5.6-6.9 \mathrm{mmol} / \mathrm{L}$ ). ${ }^{21}$ The upper glucose level for diabetes in this study was defined as fasting glucose $<7.0 \mathrm{mmol} / \mathrm{L}$.

\section{Statistical methods}

All variables were summarized using descriptive statistics such as mean, range, and frequency. Differences in continuous variables between groups (patients with psychosis and control subjects) were analyzed using analysis of covariance with age and sex as covariates. Differences in categorical variables such as sex and current smoker 
were analyzed with $\chi^{2}$ test. Categorical outcome variables with two categories such as antidiabetic treatment (no or yes) and achievement of FPG $<7 \mathrm{mmol} / \mathrm{L}$ (no or yes) were analyzed with logistic regression models with sex, age, and group (patient with psychosis or control subjects) as independent variables. In patients with psychosis who had the diagnosis of diabetes, another logistic regression analysis (stepwise forward; inclusion level, $5 \%$ ) was performed to evaluate relations between antidiabetic treatment as dependent variable and smoking status (non-smoker or smoker), fasting glucose (continuous), metabolic syndrome (no or yes), waist circumference (continuous), Global Assessment of Functioning scale (continuous), Clinical Global Impression scale (categorical), antihypertensive treatment (no or yes), lipid-lowering treatment (no or yes), duration of antipsychotic medication (months, continuous variable), and number of months in hospital during the previous year (continuous variable) as independent variables. The analyses yielded OR and $95 \% \mathrm{CI}$ as estimates of the relations. We also performed two separate regression analyses with waist circumference and fasting glucose and as outcome variables. In the first regression model waist circumference was the dependent variable and
Global Assessment of Functioning scale, Clinical Global Impression scale, fasting glucose (continuous), sex (categorical), age (continuous), duration of antipsychotic treatment and main psychiatric diagnosis (categorical) as independent variables. In the second regression model, fasting glucose was the dependent variable with the same variables as above as independent variables with the addition of waist circumference. Statistical significance was defined by $\mathrm{p} \leq 0.05$.

\section{Ethics approval}

Ethics approval for the study was obtained from the Stockholm Regional Ethics Committee. All patients and control subjects gave informed consent.

\section{RESULTS}

\section{Clinical characteristics of patients with psychosis and control subjects}

Patients who had psychosis were on average 9 years younger than control subjects. The most common psychiatric diagnosis was schizophrenia (table 1). Frequencies of smoking and mean waist circumference were greater in patients with psychosis than control subjects (table 1 ).

Table 1 Clinical characteristics of patients with psychotic disorders and control subjects*

\begin{tabular}{|c|c|c|c|}
\hline Characteristic & Patients with psychosis & Control subjects & p Value \\
\hline Number of participants & $977 \dagger$ & 3908 & \\
\hline Sex, men & $528(54)$ & $1620(41)$ & 0.001 \\
\hline Age (years) & $47(18-84)$ & $56(43-66)$ & 0.001 \\
\hline Waist circumference (cm) & $102(63-156)$ & $89(62-141)$ & 0.001 \\
\hline Men & $105(69-151)$ & $95(63-141)$ & 0.001 \\
\hline Women & $98(63-156)$ & $84(62-131)$ & 0.001 \\
\hline BMI & $29(11.2-65.8)$ & $26(16.4-53.7)$ & \\
\hline Current smoker & $397(41)$ & $666(17)$ & 0.001 \\
\hline Family history of diabetes & $184(24)$ & $956(24)$ & 0.880 \\
\hline Fasting glucose $(\mathrm{mmol} / \mathrm{L}) \ddagger$ & $5.6(3.5-20.1)$ & $5.0(2.8-14.7)$ & 0.001 \\
\hline Triglycerides $\geq 1.7 \mathrm{mmol} / \mathrm{L}$ & $400(41)$ & ND & - \\
\hline Reduced HDL levels§ & $431(44)$ & ND & - \\
\hline Metabolic syndrome & $432(45)$ & ND & - \\
\hline Antihypertensive medication & $84(9)$ & $693(18)$ & \\
\hline \multicolumn{4}{|l|}{ Psychiatric diagnosis } \\
\hline Schizophrenia & $526(54)$ & NA & - \\
\hline Psychosis NOS & $120(12)$ & NA & - \\
\hline Schizoaffective disorder & $110(11)$ & NA & - \\
\hline Delusional disorder & $63(6)$ & NA & - \\
\hline Bipolar disorder & $64(7)$ & NA & - \\
\hline Other psychiatric diagnosis & $90(9)$ & NA & - \\
\hline Antipsychotic drug & $769(79)$ & & \\
\hline Long-acting injectable & $192(24)$ & NA & \\
\hline Risperidone & $135(14)$ & NA & \\
\hline Olanzapine & $130(13)$ & $2(0.05)$ & \\
\hline Clozapine & $72(7)$ & NA & \\
\hline Aripiprazole & $58(6)$ & NA & \\
\hline
\end{tabular}

${ }^{*}$ Data reported as number, number (\%), or mean (range, minimum-maximum). †Number of patients varied due to sporadic missing information.

$\ddagger$ Patients withpsychosis and control subjects with no antidiabetic medication.

$\S \mathrm{HDL}$ level $<1.0 \mathrm{mmol} / \mathrm{L}$ (males) or $<1.3 \mathrm{mmol} / \mathrm{L}$ (females) or lipid-lowering medication.

BMI, body mass index; HDL, high-density lipoprotein; NA, not applicable; ND, not determined; NOS, not otherwise specified. 
Table 2 Antidiabetic treatment, achievement of fasting glucose $<7 \mathrm{mmol} / \mathrm{L}$ in patients with psychotic disorders and control subjects with diabetes*

\begin{tabular}{|c|c|c|c|}
\hline Glucose levels & Patients with psychosist & Control subjects & p Value \\
\hline DM diagnosis & 94 (10.0)‡ & $69(1.8)$ & $0.001 \S$ \\
\hline DM diagnosis and antidiabetic treatment & $40(42.6)$ & $38(55.1)$ & 0.114 \\
\hline Metabolic syndrome & $33(82.5)$ & ND & - \\
\hline Fasting glucose $<7.0 \mathrm{mmol} / \mathrm{L}$ & $13(32.5)$ & $12(31.6)$ & 0.931 \\
\hline DM diagnosis and no antidiabetic treatment & $54(57.4)$ & $31(44.9)$ & 0.114 \\
\hline Metabolic syndrome & $41(77.4)$ & ND & - \\
\hline Fasting glucose $<7.0 \mathrm{mmol} / \mathrm{L}$ & $32(59.3)$ & $18(58.1)$ & 0.914 \\
\hline
\end{tabular}

\section{Prevalence of prediabetes and diabetes}

Patients with psychosis without antidiabetic medication had significantly higher mean fasting glucose than control subjects (table 1). The frequency of prediabetes (fasting glucose level, $6.1-6.9 \mathrm{mmol} / \mathrm{L} ; 9.9 \%$ ) and diabetes $(10.0 \%)$ were similar in patients with psychosis and more frequent in patients with psychosis than control subjects (tables 2 and 3). The difference between patients with psychosis and control subjects in prevalence of prediabetes was greatest in participants aged 50-59 years (figure 1). The same most pronounced difference in younger age intervals was also observed for fasting glucose level $5.6-6.0 \mathrm{mmol} / \mathrm{L}$ (figure 2). Furthermore, 64 (77\%) patients with psychosis with fasting glucose $6.1-6.9 \mathrm{mmol} / \mathrm{L}$ and 103 $(65 \%)$ patients with psychosis with fasting glucose 5.6$6.0 \mathrm{mmol} / \mathrm{L}$ also satisfied the criteria for metabolic syndrome (table 3 ).

\section{Antidiabetic medication}

There was no significant difference in the frequency of antidiabetic medication in patients with psychosis and control subjects with diagnosed diabetes (table 2). There was no significant difference in achievement of FPG $<7 \mathrm{mmol} / \mathrm{L}$ between patients with psychosis and control subjects (table 2). Most patients with psychosis and control subjects with diabetes were on monotherapy with metformin, and 32 participants (patients with psychosis and control subjects combined; $41 \%$ ) were taking insulin (table 4). Patients with psychosis taking antidiabetic medication more frequently used insulin and less frequently used a combination of oral antidiabetic drugs than control subjects (table 4). Owing to the small sample size, these differences were not tested for significance.

\section{Factors associated with antidiabetic treatment}

Logistic regression (stepwise forward) to evaluate potential factors associated with having antidiabetic medical treatment when diagnosed with diabetes and psychosis showed that lipid-lowering medication (OR 6.74; 95\% CI 1.88 to 24.13 ) and high fasting glucose (OR 1.24; $95 \%$ CI 1.01 to 0.72 ) were associated with antidiabetic treatment. No other variables that were entered in the logistic regression model were significantly associated with antidiabetic medical treatment.

\section{Differences between psychosis diagnoses}

Patients with schizophrenia were analyzed separately because they comprised $54 \%$ of the patients with psychosis (data not shown). There was a small but significant difference in mean FPG, with higher values for patients

Table 3 Increased fasting glucose in patients and control subjects without diabetes*

\begin{tabular}{llll}
\hline Glucose levels & Patients with psychosis $†$ & Control subjects & p Value \\
\hline No DM diagnosis & $854(90.1)$ & $3839(98.2)$ & $0.001 \ddagger$ \\
Fasting glucose $\geq 7.0 \mathrm{mmol} / \mathrm{L}$ & $43(5.0)$ & $41(1.1)$ & $\mathrm{ND}$ \\
$\quad$ Metabolic syndrome & $32(76.2)$ & $149(3.8)$ & - \\
Fasting glucose $6.1-6.9 \mathrm{mmol} / \mathrm{L}$ & $87(10.2)$ & $\mathrm{ND}$ & $0.001 \ddagger$ \\
Metabolic syndrome & $64(77.1)$ & $355(9.1)$ & - \\
Fasting glucose $5.6-6.0 \mathrm{mmol} / \mathrm{L}$ & $160(18.7)$ & $\mathrm{ND}$ & $0.001 \ddagger$ \\
Metabolic syndrome & $103(65.2)$ & & - \\
\hline
\end{tabular}

${ }^{*}$ Data reported as number (\%).

†Number of patients varied due to sporadic missing information.

$\neq p$ Value from logistic regression models controlling for differences in sex and age.

DM, diabetes mellitus; ND, not determined. 
Figure 1 Relation between age and impaired fasting glucose (fasting glucose, 6.1-6.9 mmol/L) in patients with psychotic disorders and control subjects.
Fasting Glucose $6.1-6.9 \mathrm{mmol}$

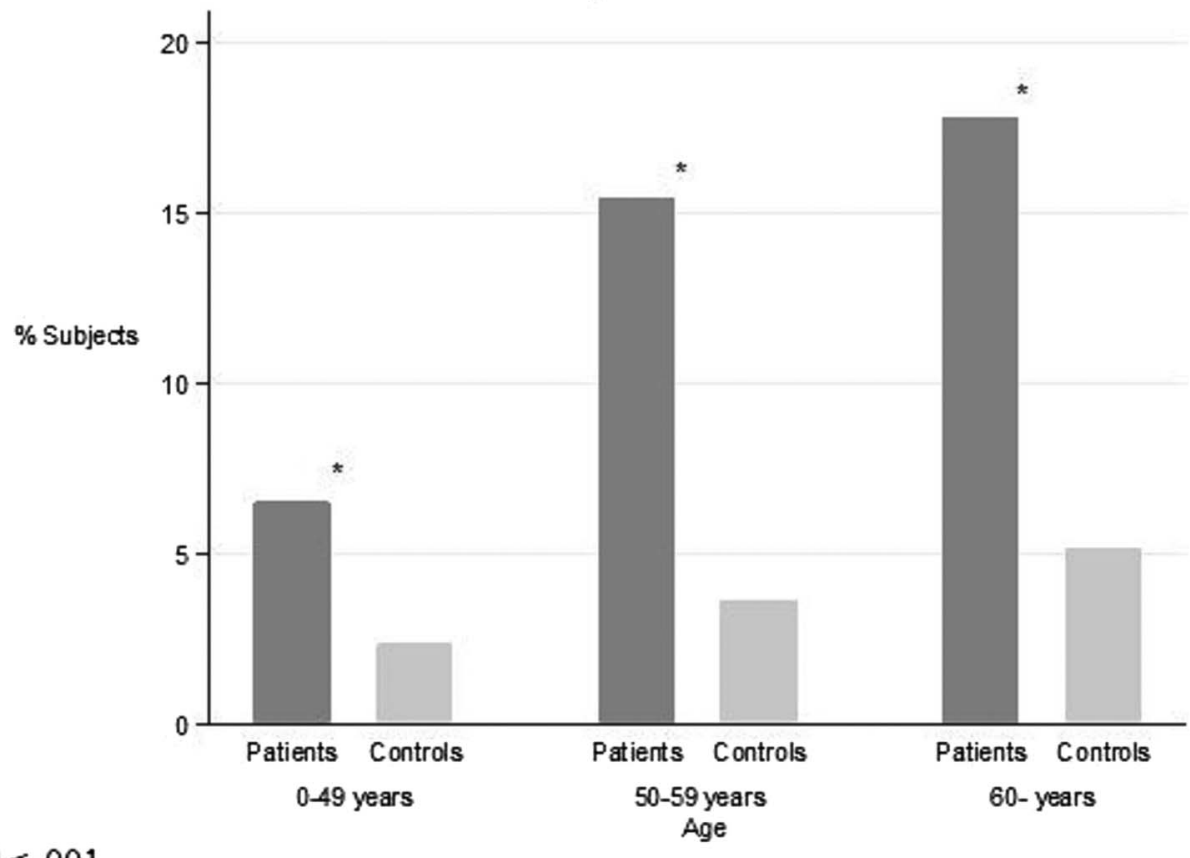

${ }^{8} P \leq .001$.

with schizophrenia than patients who had other diagnoses of psychosis. We observed no significant difference in frequency of diabetes diagnosis or use of antidiabetic medication between patients with schizophrenia and other patients with psychosis. Logistic regression analysis performed separately for patients with schizophrenia and diabetes showed a positive association between lipid-lowering medication and antidiabetic treatment. Also, a negative association was observed between duration of antipsychotic treatment and antidiabetic treatment (OR 0.88; $95 \%$ CI 0.79 to 0.97 ).
Associations with waist circumference and fasting glucose Potential factors associated with waist circumference and fasting glucose were studied in two regression models (data not shown). There was a significant positive association between duration of antipsychotic treatment, high fasting glucose, being male, and increased waist circumference. The other independent variables entered showed no significant association. Increased waist circumference and age were the only independent variables with a positive significant association with increased fasting glucose.
Figure 2 Relation between age and impaired fasting glucose (fasting glucose, $5.6-6.0 \mathrm{mmol} / \mathrm{L}$ ) in patients with psychotic disorders and control subjects.

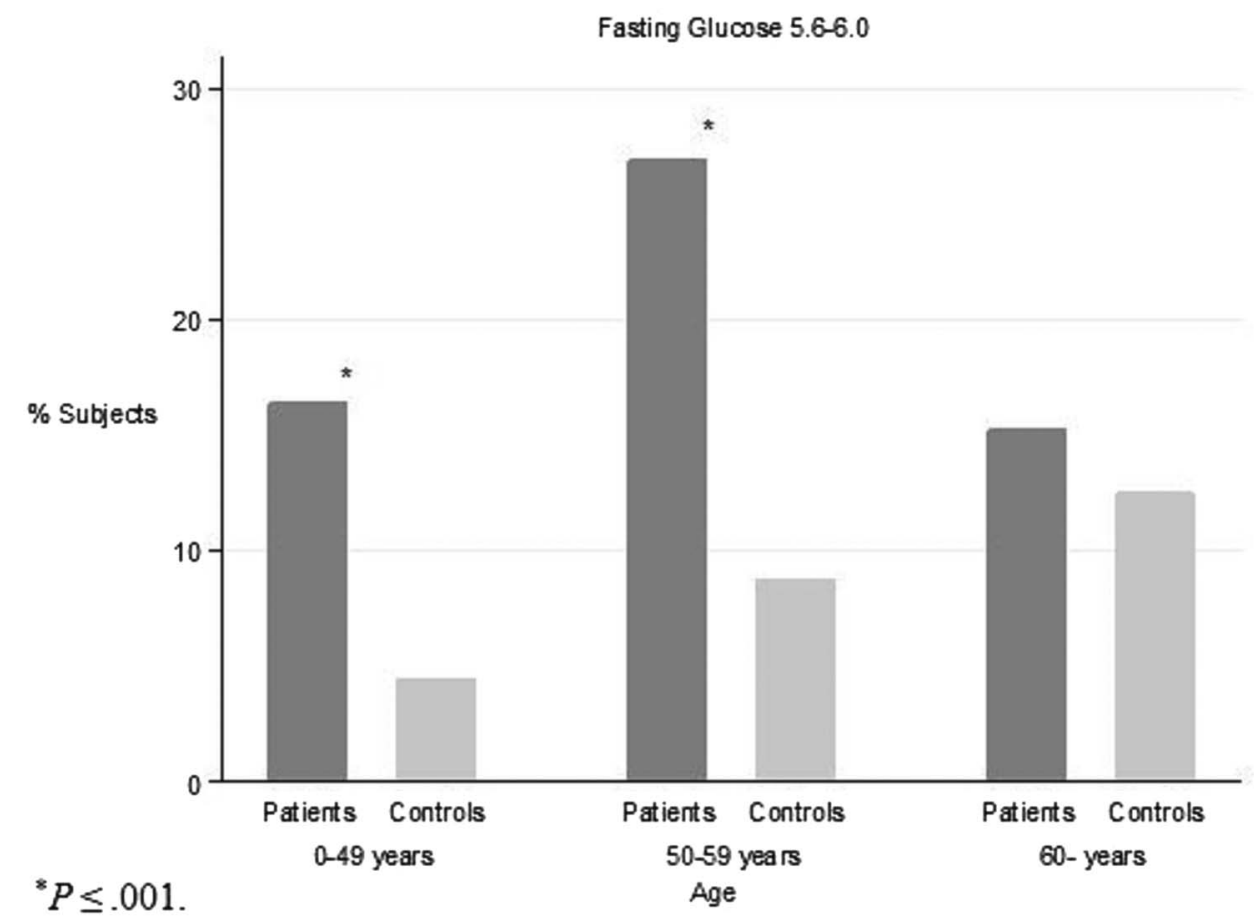


Table 4 Antidiabetic drugs, fasting glucose levels, and achievement of fasting glucose $<7 \mathrm{mmol} / \mathrm{L}$ in patients with psychotic disorders and control subjects*

\begin{tabular}{|c|c|c|c|c|c|c|}
\hline \multirow[b]{2}{*}{ Antidiabetic drugs } & \multirow{2}{*}{$\begin{array}{l}\text { Patients with } \\
\text { psychosis }\end{array}$} & \multicolumn{2}{|c|}{ Fasting glucose (mmol/L) } & \multirow{2}{*}{$\begin{array}{l}\text { Control } \\
\text { subjects } †\end{array}$} & \multicolumn{2}{|c|}{ Fasting glucose (mmol/L) } \\
\hline & & Mean (range) & $<7.0 \mathrm{mmol} / \mathrm{L}$ & & Mean (range) & $<7.0 \mathrm{mmol} / \mathrm{L}$ \\
\hline Number of participants & 40 & & & 38 & & \\
\hline One oral antidiabetic drug & $19(48)$ & $8.5(4.9-15.4)$ & $6(32)$ & $18(47)$ & $7.7(6.2-11.1)$ & $5(28)$ \\
\hline Metformin & $13(68)$ & $7.4(4.9-12.4)$ & $6(46)$ & $11(61)$ & $7.6(6.2-9.7)$ & $2(18)$ \\
\hline Glibenclamide & $4(21)$ & $11.3(7.2-15.4)$ & $0(0)$ & $5(28)$ & $7.5(6.3-11.1)$ & $3(60)$ \\
\hline Otherł & $2(11)$ & $10.2(8.2-12.1)$ & $0(0)$ & $2(11)$ & $8.9(7.6-10.1)$ & $0(0)$ \\
\hline$\geq$ Two oral antidiabetic drugs & $3(8)$ & $8.5(6.3-12.7)$ & $2(67)$ & $6(16)$ & $9.1(7.7-10.0)$ & $0(0)$ \\
\hline Insulin & $18(45)$ & $9.4(3.2-23.6)$ & $5(28)$ & $14(37)$ & $8.2(3.9-14.0)$ & $7(50)$ \\
\hline
\end{tabular}

\section{DISCUSSION}

The main finding of this study of patients with psychosis in Sweden was the markedly increased frequency of prediabetes, defined as FPG $6.1-6.9 \mathrm{mmol} / \mathrm{L}$, in patients with psychosis compared with control subjects. In participants aged 50-59 years, prediabetes was observed in $15 \%$ of patients with psychosis but less than $5 \%$ of the control subjects. In addition, $64(77 \%)$ patients with psychosis who had prediabetes satisfied the criteria for metabolic syndrome.

In Sweden, specialized psychosis clinics are responsible for the treatment of the vast majority of outpatients who have chronic psychotic disorders. As part of general health screening, all patients with psychosis in regular clinical treatment from several clinics were asked to participate in the study. Therefore, the results may be generalizable to the entire population of patients with psychosis. The control sample was population-based, but it was refined for family history of diabetes, and individuals with diabetes at baseline were excluded. The control sample in this study included only patients with diabetes diagnosed between baseline and follow-up. Thus, the patients with psychosis and the control subjects were not comparable regarding occurrence of diabetes. However, since control subjects at baseline and patients with psychosis were similar in age, the estimated prevalence of diabetes (known and newly diagnosed at baseline among control subjects) could be compared with the prevalence in patients with psychosis. The patients with psychosis and diabetes were likely to have had diabetes longer than control subjects and included more patients with type 1 diabetes. It was not possible to differentiate between type 1 and type 2 diabetes. Moreover, patients and controls did not go through exactly the same examinations. Patients had a lipid profiling done and controls went through an oral glucose tolerance test but not vice versa. Thus, it was not possible to assess the control subjects for metabolic syndrome, and controls were more thoroughly screened for diabetes. Furthermore, controls were not screened for severe mental illnesses which could bias the results. However, only four control subjects had recorded use of antipsychotic medication, two with olanzapine and two with zuclopenthixol. The control subjects at follow-up were 9 years older than the patients with psychosis. This difference was statistically controlled for in the comparative analysis, because higher age could have caused an overestimate of the prevalence of diabetes and prediabetes. Even when controlling for the age difference, caution is warranted when interpreting results.

Diabetes prevalence was 2.7-fold higher in patients with psychosis $(10.0 \%)$ than control subjects at baseline $(3.7 \%)$. Cases were on average 3 years younger than control subjects in this comparison. The high prevalence of diabetes among patients with psychosis in this study is consistent with previous findings that showed a twofold to threefold increase in patients with schizophrenia. ${ }^{22} 23$ In patients with psychosis, $432(45 \%)$ satisfied the criteria for metabolic syndrome. This was slightly higher than the results of a previous study of patients with psychosis who were an average 10 years younger than our sample. ${ }^{24}$

When comparing diabetes treatment and outcome between patients with psychosis and control subjects, it is important that all persons with diabetes at baseline in the control group were excluded from further studies. The use of FPG $<7 \mathrm{mmol} / \mathrm{L}$ to assess antidiabetic treatment instead of glycated hemoglobin could possibly bias the results. It is possible, although not confirmed in the literature, that a larger proportion of patients compared with controls did not comply with instructions to be fasting when the blood sample was drawn. The underestimation of diabetes prevalence in control subjects compared with patients with psychosis will not be the case regarding impaired fasting glucose, since these control subjects were not excluded at baseline. The prevalence of prediabetes, defined as FPG 6.1$6.9 \mathrm{mmol} / \mathrm{L}$, and impaired fasting glucose, defined as FPG 5.6-6.0 mmol/L, was higher in patients with psychosis than control subjects. Furthermore, most patients with psychosis with diabetes or fasting glucose $\geq 5.6 \mathrm{mmol} / \mathrm{L}$ satisfied the criteria for the metabolic syndrome. The metabolic syndrome is a condition with an increased risk of developing diabetes. ${ }^{25}$ The higher 
prevalence of prediabetes or impaired fasting glucose (FPG $6.1-6.9$ or $5.7-6.0 \mathrm{mmol} / \mathrm{L}$ ) was most pronounced in younger age groups comparing patients with psychosis to control subjects (figures 1 and 2).

The results from the regression model in this study indicated that the degree of somatic illness was more important than degree of psychiatric illness with respect to medical antidiabetic treatment. Thus, when patients with psychosis were identified with diabetes, the factors associated with antidiabetic treatment were high fasting glucose and presence of lipid-lowering medication. These findings are not surprising since the Swedish clinical guidelines recommend that all patients with diabetes and LDL $>2.5$ and/or cholesterol $>5 \mathrm{mmol} / \mathrm{L}$ should be treated with lipid-lowering medication (statins).

This study was successful in identifying not only the extent of diabetes but also the prevalence dysglycemia in schizophrenia in a fair sized sample of representative schizophrenic population treated in naturalistic settings. The high proportion of patients with psychosis with impaired fasting glucose suggest that a prospective study may be justified to evaluate the progression of prediabetes to type 2 diabetes in patients with psychosis and the potential preventive effects on diabetes by lifestyle programmes targeting prediabetes. It also would be important to perform prospective studies of medical treatment with metformin or newer agents such as glucagon-like polypeptide-1 (GLP-1) analogs in patients with psychosis and prediabetes, to assess the effects in preventing diabetes. In the general population, both metformin and intensive lifestyle interventions may reduce the progression from prediabetes to type 2 diabetes. ${ }^{12}{ }^{26}$ There are also newer treatment options such as GLP-1 analogs to manage overweight, prediabetes, and type 2 diabetes. ${ }^{27}$ However, most of these newer agents lack safety data and data in reduction of mortality and morbidity compared with metformin. Individuals who are at high risk for developing diabetes, based on models considering metabolic parameters, may benefit most from treatment with metformin when diagnosed with prediabetes. ${ }^{28}$ It is unknown whether this is true for patients with psychosis, but metformin may reduce antipsychotic weight gain. ${ }^{29}$ Intensive treatment may be indicated for patients with psychosis who have fasting glucose $>6.0 \mathrm{mmol} / \mathrm{L}$, but our data also indicate that it may be advisable to include patients with psychosis who have lower fasting glucose levels, especially when they satisfy the criteria for metabolic syndrome.

In summary, diabetes and prediabetes are common in patients with psychosis and are a major public health problem. Patients with psychosis at risk for diabetes can be identified by diagnosing prediabetes several years before the onset of diabetes. Reduced progression to diabetes should be an important treatment goal, and prospective randomized treatment studies should be performed with lifestyle interventions and pharmacological treatment. Responsibility for diagnosis and treatment of hyperglycemia for patients with psychosis should be clearly defined as a specific task for general healthcare, as in the general population, so that preventive treatment programmes may be started when patients with psychosis satisfy treatment criteria.

Author affiliations

${ }^{1}$ Department of Clinical Neuroscience, Karolinska Institutet, Stockholm, Sweden

${ }^{2}$ Department of Adult Psychiatry, PRIMA Barn och Vuxenpsykiatri AB, Stockholm, Sweden

${ }^{3}$ Department of Neurobiology, Care Sciences and Society, Centre of Family

Medicine, Karolinska Institutet, Stockholm, Sweden

${ }^{4}$ Center for Molecular Medicine, Karolinska University Hospital, Stockholm, Sweden

${ }^{5}$ Department of Clinical Sciences, Karolinska Institutet, Danderyd University Hospital, Stockholm, Sweden

${ }^{6}$ Department of Psychiatry, Tiohundra AB, Norrtälje, Sweden

${ }^{7}$ Department of Medicine Solna, Centre for Pharmacoepidemiology, Karolinska Institutet, Stockholm, Sweden

${ }^{8}$ Department of Neuroscience, Psychiatry, Uppsala University, Uppsala, Sweden

${ }^{9}$ Department of Molecular Medicine and Surgery, Karolinska Institutet, Stockholm, Sweden

Contributors EO has made substantial contributions to the analysis and interpretation of data for the work, drafting the work and revising it critically for important intellectual content, and final approval of the version to be published. JW, DSH, SVE, RB, EJ, JR, and AB have made substantial contributions to the analysis and interpretation of data for the work, revising it for important intellectual content, and final approval of the version to be published. GE, AH, MS, C-GÖ, and UÖ have made substantial contributions to the conception or design of the work, acquisition of data, revision process for important intellectual content, and final approval of the version to be published. All the authors agree to be accountable for all aspects of the work in ensuring that questions related to the accuracy or integrity of any part of the work are appropriately investigated and resolved.

Funding This work received grant support from PRIMA Child and Adult Psychiatry Inc and from Söderström-Königska Foundation.

Competing interests UÖ received funding for attending courses from Janssen-Cilag and has research collaboration with Lundbeck A/S.

Ethics approval Stockholm Regional Ethics Committee.

Provenance and peer review Not commissioned; externally peer reviewed.

Data sharing statement No additional data are available.

Open Access This is an Open Access article distributed in accordance with the Creative Commons Attribution Non Commercial (CC BY-NC 4.0) license, which permits others to distribute, remix, adapt, build upon this work noncommercially, and license their derivative works on different terms, provided the original work is properly cited and the use is non-commercial. See: http:// creativecommons.org/licenses/by-nc/4.0/

\section{REFERENCES}

1. Brown S. Excess mortality of schizophrenia. A meta-analysis. $\mathrm{Br} \mathrm{J}$ Psychiatry 1997;171:502-8.

2. Osby U, Correia N, Brandt L, et al. Mortality and causes of death in schizophrenia in Stockholm county, Sweden. Schizophr Res 2000;45:21-8.

3. Saha S, Chant D, McGrath J. A systematic review of mortality in schizophrenia: is the differential mortality gap worsening over time? Arch Gen Psychiatry 2007;64:1123-31.

4. Newcomer JW, Hennekens $\mathrm{CH}$. Severe mental illness and risk of cardiovascular disease. JAMA 2007;298:1794-6.

5. Osby U, Hammar N, Brandt L, et al. Time trends in first admissions for schizophrenia and paranoid psychosis in Stockholm County, Sweden. Schizophrenia Res 2001;47:247-54.

6. Crump C, Winkleby MA, Sundquist K, et al. Comorbidities and mortality in persons with schizophrenia: a Swedish national cohort study. Am J Psychiatry 2013;170:324-33. 
7. Haffner SM, Lehto S, Rönnemaa T, et al. Mortality from coronary heart disease in subjects with type 2 diabetes and in nondiabetic subjects with and without prior myocardial infarction. $N$ Engl J Med 1998;339:229-34.

8. McEvoy JP, Meyer JM, Goff DC, et al. Prevalence of the metabolic syndrome in patients with schizophrenia: baseline results from the Clinical Antipsychotic Trials of Intervention Effectiveness (CATIE) schizophrenia trial and comparison with national estimates from NHANES III. Schizophr Res 2005;80:19-32.

9. Newcomer JW. Second-generation (atypical) antipsychotics and metabolic effects: a comprehensive literature review. CNS Drugs 2005;19(Suppl 1):1-93.

10. Osby U, Olsson E, Edman G, et al. Psychotic disorder is an independent risk factor for increased fasting glucose and waist circumference. Nord J Psychiatry 2014;68: 215-18.

11. Bushe $\mathrm{C}$, Holt R. Prevalence of diabetes and impaired glucose tolerance in patients with schizophrenia. $\mathrm{Br} J$ Psychiatry Suppl 2004;47:S67-71

12. Merlotti C, Morabito A, Pontiroli AE. Prevention of type 2 diabetes; a systematic review and meta-analysis of different intervention strategies. Diabetes Obes Metab 2014;16:719-27.

13. American Psychiatric Association. Diagnostic and Statistical Manual of Mental Disorders. 4th edn. Text Revision. Washington DC: American Psychiatric Association, 2000.

14. Eriksson AK, Ekbom A, Granath F, et al. Psychological distress and risk of pre-diabetes and type 2 diabetes in a prospective study of Swedish middle-aged men and women. Diabet Med 2008;25:834-42.

15. Aas IH. Guidelines for rating Global Assessment of Functioning (GAF). Ann Gen Psychiatry 2011;10:2.

16. Busner J, Targum SD. The clinical global impressions scale: applying a research tool in clinical practice. Psychiatry (Edgmont) 2007:4:28-37.

17. Alberti KG, Zimmet P, Shaw J; IDF Epidemiology Task Force Consensus Group. The metabolic syndrome-a new worldwide definition. Lancet 2005;366:1059-62.
18. Alberti KG, Zimmet P, Shaw J. Metabolic syndrome-a new world-wide definition. A consensus statement from the International Diabetes Federation. Diabet Med 2006;23:469-80.

19. Eckel RH, Alberti KG, Grundy SM, et al. The metabolic syndrome. Lancet 2010;375:181-3.

20. Alberti KG, Zimmet PZ. Definition, diagnosis and classification of diabetes mellitus and its complications. Part 1: diagnosis and classification of diabetes mellitus provisional report of a $\mathrm{WHO}$ consultation. Diabet Med 1998;15:539-53.

21. American Diabetes Association. Diagnosis and classification of diabetes mellitus. Diabetes Care 2007;30(Suppl 1):S42-7.

22. De Hert M, van Winkel R, Van Eyck D, et al. Prevalence of diabetes, metabolic syndrome and metabolic abnormalities in schizophrenia over the course of the illness: a cross-sectional study. Clin Pract Epidemiol Ment Health 2006;2:14.

23. Dixon L, Weiden P, Delahanty J, et al. Prevalence and correlates of diabetes in national schizophrenia samples. Schizophr Bull 2000;26:903-12.

24. De Hert MA, van Winkel R, Van Eyck D, et al. Prevalence of the metabolic syndrome in patients with schizophrenia treated with antipsychotic medication. Schizophr Res 2006;83:87-93.

25. Cheal KL, Abbasi F, Lamendola C, et al. Relationship to insulin resistance of the adult treatment panel III diagnostic criteria for identification of the metabolic syndrome. Diabetes 2004;53:1195-200.

26. Knowler WC, Fowler SE, Hamman RF, et al; Diabetes Prevention Program Research Group. 10-year follow-up of diabetes incidence and weight loss in the Diabetes Prevention Program Outcomes Study. Lancet 2009;374:1677-86.

27. Cahn A, Miccoli R, Dardano A, et al. New forms of insulin and insulin therapies for the treatment of type 2 diabetes. Lancet Diabetes Endocrinol 2015;3:638-52.

28. Sussman JB, Kent DM, Nelson JP, et al. Improving diabetes prevention with benefit based tailored treatment: risk based reanalysis of Diabetes Prevention Program. BMJ 2015;350:h454.

29. Wu RR, Zhao JP, Jin $\mathrm{H}$, et al. Lifestyle intervention and metformin for treatment of antipsychotic-induced weight gain: a randomized controlled trial. JAMA 2008;299:185-93. 